\title{
Biomarkers in clinical management of pulmonary hypertension: has the emperor no clothes? A call for action
}

\author{
Gian Luca lannuzzi ${ }^{1}$, Michele D'Alto², Roberto Formisano ${ }^{1}$ \& Mauro Maniscalco*,3 \\ ${ }^{1}$ Cardiac Rehabilitation Unit, Maugeri Clinical \& Scientific Institutes ICSR, Telese (BN), Italy \\ ${ }^{2}$ Pulmonary Hypertension Centre, L. Vanvitelli University of Campania-Monaldi Hospital, Naples, Italy \\ ${ }^{3}$ Pulmonary Rehabilitation Unit, Maugeri Clinical \& Scientific Institutes ICSR, Telese (BN), Italy \\ *Author for correspondence: Tel.: +39 082490 9350; Fax: +39 082490 9350; mauromaniscalco@hotmail.com
}

“Clearly, being an invasive procedure with a given share of complications, right heart catheterization cannot be repeated indefinitely in $\mathrm{PH}$ patients. No doubt that echocardiography can warrant a paramount contribution to this issue"

First draft submitted: 27 December 2018; Accepted for publication: 15 January 2019; Published online: 19 March 2019

Keywords: biomarkers $\bullet$ inflammation $\bullet$ lung $\bullet$ rehabilitation

Pulmonary hypertension $(\mathrm{PH})$ is a manifold, lethal disease with various etiologies and subtypes, all sharing a common factor: the milestone of diagnosis and clinical management is right heart catheterization, the gold standard for both quantification of $\mathrm{PH}$ and correct identification of its cause [1]. Clearly, being an invasive procedure with a given share of complications, right heart catheterization cannot be repeated indefinitely in $\mathrm{PH}$ patients. No doubt that echocardiography can warrant a paramount contribution to this issue [2], although echocardiographic assessment of $\mathrm{PH}$ should be considered as an estimate, rather than an exact measurement. Therefore, many efforts have been made in order to assess the role played by biomarkers in PH, overcoming the hurdle of studying patients affected by such a rare and deadly disease.

$\mathrm{PH}$ European guidelines divide biomarkers involved in $\mathrm{PH}$ progression into distinguished groups: vascular dysfunction, inflammation, myocardial stress, low cardiac output and/or tissue hypoxia or organ impairment [1]. Similarly, Pezzuto et al. grouped circulating biochemical markers based on their role in: neurohormonal activation, myocardial injury, systemic inflammation or oxidative stress, vascular remodeling, end-organ failure, transcriptional regulation and gene expression [3]. Among these, biomarkers of systemic inflammation and altered immune response (namely, circulating chemokines and cytokines [4], metabolomics factors [5] and volatile substances) have been shown to play a pivotal role. These have also been investigated as possible therapeutic targets, linking gene expression and mutations to the clinical world, as in the case of TNF- $\alpha$ [6]. Yet, just for BNP and NT-proBNP a prominent role in risk assessment and clinical management of arterial $\mathrm{PH}$ patients has been acknowledged until now [1]. Actually, as a marker of myocardial stress and myocardial dysfunction, BNP/NT-proBNP (even if with quite variable and low specificity for $\mathrm{PH}$ ) is the only biochemical marker acknowledged for both the daily clinic and for research studies, being part of the multidimensional management of $\mathrm{PH}$ patients that current guidelines recommend [1].

Even if biomarkers are well known to be changed in $\mathrm{PH}$, their utilization at the patient's bedside is marginal. Indeed, it still remains to be established whether these factors play a own role in the complex pathophysiologic cascade of $\mathrm{PH}$, or if they should rather be considered as mere markers for the progression of disease.

BNP/NT-proBNP levels correlate with myocardial dysfunction, but they are not specific for $\mathrm{PH}$, because they can be elevated in almost any heart disease. BNP/NT-proBNP levels tend to have a high variability and should be interpreted in the clinical context. There are no clear advantages in using BNP versus NT-proBNP [7]. Nevertheless, BNP appears to have a stronger correlation with pulmonary hemodynamics and is less affected by kidney function, while NT-proBNP seems to be a stronger predictor of prognosis [7]. 
Data from the registry to evaluate early and long-term pulmonary arterial hypertension disease management (REVEAL) [8] showed that changes in a multiparametric score including BNP/NT-pro-BNP occur in most patients with arterial $\mathrm{PH}$ over a 12-month period and are predictive of survival. Thus, serial risk score assessments can identify changes in disease course that may warrant treatment adjustments.

Recently, three large European studies [9-11] suggested using comprehensive risk assessment scores aimed at reaching a low-risk profile in arterial $\mathrm{PH}$. All three studies confirmed the prognostic role of BNP/NT-pro-BNP in a multi-parametric approach for defining the prognosis in arterial $\mathrm{PH}$.

Regardless of all of these caveats, clinicians managing arterial PH (or even groups II-IV PH, which are PH depending on left heart disease, lung disease and pulmonary embolism, respectively [1]) generally use few biomarkers, and - to the best of our knowledge - almost exclusively as secondary tools. Consequently, a main issue seems to be focusing research on the changes in biochemical markers occurring during the frequent episodes of clinical worsening and increase in mean pulmonary arterial pressure (PAP) that these kinds of patients often experience, whereas it should be to find out a number of factors that are able to predict these kinds of events.

The ideal biomarker should of course show good sensitivity, specificity and reproducibility [12]. It should also be safe, cheap, easily accessible, with changes wide enough to be measured, it should mirror clinical course, response to treatments and, mainly, the pressure regimen of the pulmonary circle itself. In one word: reliable, but probably unattainable. Then, what direction should we search for?

With the aim to help clinicians in making decisions in the follow-up of $\mathrm{PH}$ patients, a cluster of biomarkers has been recently proposed: in their study Rhodes et al. [13] found a group of nine circulating proteins to be related with survival in arterial PH, improving the predictive power of NT-proBNP by itself. Their results are of high interest as the studied proteins are all involved in biological pathways known to be changed in PH (i.e., vascular injury, endothelial function, inflammation state, immune response and pro-thrombotic state) and the named nine circulating proteins show a higher predictive value when assessed together, with respect to their use as single items [13]. Unfortunately, measuring the circulating levels of nine proteins implies resources and technologies not largely available.

Moving from the consideration that aldosterone and Galectin-3 are changed in left ventricular failure, Calvier et al. [14] explored their role in a classic right-side heart failure model as arterial PH, showing this tandem (already known to be related with inflammation, fibrosis and, in turn, vascular remodeling) to be increased in such patients.

Interestingly, various biomarkers derived from everyday routine laboratory parameters, for example, uric acid, C-reactive protein, high-density lipoproteins [3], creatinine, bilirubin, blood cholesterol [7,15,16], red cell distribution width [17], D-dimer, troponin and natriuretic peptides [18,19] have proven to be singularly involved in the complex galaxia of $\mathrm{PH}$, including fields as heart or other organ dysfunction, oxidative stress, endothelial dysfunction $[18,19]$ and are recommended to be regularly checked in PH patients [1].

In other medical fields, serious diseases are currently diagnosed based on composite criteria arranged in validated clinical tools: JONES criteria for rheumatic fever or the signs observed in metabolic syndrome are a couple of the numerous examples confirming this statement. Accordingly, in atrial fibrillation, a lifesaving treatment as oral anticoagulation is given on the basis of two scores, such as $\mathrm{CHA}_{2} \mathrm{DS}_{2}$ VASC and HAS-BLED, that are strong predictors of cerebrovascular events and hemorrhagic risk, respectively [20].

Indeed, both these two risk scores are mainly made up of clinical items (e.g., age, hypertension and heart failure, but also INR lability, liver and renal function, etc.), rather than laboratory or biochemical markers. Yet the key concept is to observe how much the validation of these two clinical tools has improved the management of patients of all ages with atrial fibrillation all over the world.

Why not try to find out a composite score based on biochemical markers and related to the clinical course of $\mathrm{PH}$ ? This is certainly not the first time this hypothesis is formulated, but we believe the knowledge about $\mathrm{PH}$ is now deep enough and the time is ready for facing this major challenge.

For example, von Willebrand factor, cholesterol levels and NT-proBNP have already been considered as a whole, but in a population retrospectively selected by another clinical trial with other purposes and with several inclusion/exclusion criteria [16].

The main issues to manage within follow-up of $\mathrm{PH}$ patients seem to be to predict clinical worsening (or at least to get a very early diagnosis of it) and to acknowledge the response to treatment. This kind of clinical tool should aim at a different target from supplying an indirect measure of mean PAP: for that we already have echocardiography, which is able to fairly assess systolic, diastolic, mean PAP [2], as well as (with less accuracy) wedge pressures, pulmonary vascular resistances, right ventricle filling pressures [21]. It should also be noted that the various 
groups of PH largely differ as signs, symptoms and clinical picture as a whole. It will not be easy to identify items appropriate, for example for both group I and group III PH patients. Yet, if such a clinical score could ever be available for physicians dealing with $\mathrm{PH}$ patients, and if its robustness and feasibility in clinical settings were good enough, the management of these frail patients could be predictably improved. Support for this call also derives from the fact that standardization generally follows the adoption of a clinical score, like we hypothesized, could allow an easier collection and analysis of patients' data, which is always a key issue - largely in rare diseases like in most forms of $\mathrm{PH}$ [1].

In conclusion, we argue a call for identifying a clinical score, including biochemical markers (among these, BNP and NT-proBNP) in PH to be highly reasonable, and that this tool could conceivably warrant a progress in the management of patients with arterial PH (i.e., group I), including many of the other $\mathrm{pH}$ patient groups.

\section{Financial \& competing interests disclosure}

The authors have no relevant affiliations or financial involvement with any organization or entity with a financial interest in or financial conflict with the subject matter or materials discussed in the manuscript. This includes employment, consultancies, honoraria, stock ownership or options, expert testimony, grants or patents received or pending, or royalties.

No writing assistance was utilized in the production of this manuscript.

\section{References}

Papers of special note have been highlighted as: $\bullet$ of interest; $\bullet \bullet$ of considerable interest

1. Galie N, Humbert M, Vachiery JL et al. 2015 ESC/ERS Guidelines for the diagnosis and treatment of pulmonary hypertension: The Joint Task Force for the Diagnosis and Treatment of Pulmonary Hypertension of the European Society of Cardiology (ESC) and the European Respiratory Society (ERS): Endorsed by: Association for European Paediatric and Congenital Cardiology (AEPC), International Society for Heart and Lung Transplantation (ISHLT). Eur. Heart J. 37(1), 67-119 (2016).

- Last guidelines published about pulmonary hypertension, essential to anyone dealing with this kind of patients.

2. Krzeminska-Pakula M, Binikowski Z, Marszal-Marciniak M, Rafalska K. Doppler echocardiography in the estimation of systolic, diastolic and mean pulmonary artery pressure. Acta Cardiol. 43(3), 329-334 (1988).

3. Pezzuto B, Badagliacca R, Poscia R et al. Circulating biomarkers in pulmonary arterial hypertension: update and future direction. $J$. Heart Lung Transplant. 34(3), 282-305 (2015).

- Broad, complete, up-to-date review of biochemical markers involved in pulmonary arterial hypertension, divided by pathophysiological area.

4. Rabinovitch M, Guignabert C, Humbert M, Nicolls MR. Inflammation and immunity in the pathogenesis of pulmonary arterial hypertension. Circ. Res. 115(1), 165-175 (2014).

5. Maniscalco M, Cutignano A, Paris D et al. Metabolomics of exhaled breath condensate by nuclear magnetic resonance spectroscopy and mass spectrometry: a methodological approach. Curr. Med. Chem. doi:10.2174/0929867325666181008122749 (2018) (Epub ahead of print).

6. Hurst LA, Dunmore BJ, Long L et al. TNFalpha drives pulmonary arterial hypertension by suppressing the BMP type-II receptor and altering NOTCH signalling. Nat. Commun. 8, 14079 (2017).

7. Leuchte HH, El Nounou M, Tuerpe JC et al. N-terminal pro-brain natriuretic peptide and renal insufficiency as predictors of mortality in pulmonary hypertension. Chest 131(2), 402-409 (2007).

8. Benza RL, Miller DP, Foreman AJ et al. Prognostic implications of serial risk score assessments in patients with pulmonary arterial hypertension: a Registry to Evaluate Early and Long-Term Pulmonary Arterial Hypertension Disease Management (REVEAL) analysis. J. Heart Lung Transplant. 34(3), 356-361 (2015).

- Trial confirming BNP/NT-pro-BNP as a key biomarker in arterial pulmonary hypertension, supplying to its low specificity by including it in a multiparametric clinical score.

9. Boucly A, Weatherald J, Savale L et al. Risk assessment, prognosis and guideline implementation in pulmonary arterial hypertension. Eur. Respir. J. 50(2), pii:1700889 (2017).

10. Hoeper MM, Kramer T, Pan Z et al. Mortality in pulmonary arterial hypertension: prediction by the 2015 European pulmonary hypertension guidelines risk stratification model. Eur. Respir. J. 50(2), (2017).

11. Kylhammar D, Kjellstrom B, Hjalmarsson C et al. A comprehensive risk stratification at early follow-up determines prognosis in pulmonary arterial hypertension. Eur. Heart J. 39(47), 4175-4181 (2018).

12. Maniscalco M, Paris D, Carone M, Spanevello A, Vitacca M, Motta A. Is there a role for biomarkers in pulmonary rehabilitation? Biomark. Med. 12(10), 1069-1072 (2018).

13. Rhodes CJ, Wharton J, Ghataorhe P et al. Plasma proteome analysis in patients with pulmonary arterial hypertension: an observational cohort study. Lancet Resp. Med. 5(9), 717-726 (2017). 
- Noteworthy paper, assessing the value of a cluster of circulating proteins in predicting mortality in arterial pulmonary hypertension patients. The value of the study is in the addictive predicting value of the biomarkers when considered as a whole.

14. Calvier L, Legchenko E, Grimm L et al. Galectin-3 and aldosterone as potential tandem biomarkers in pulmonary arterial hypertension. Heart 102(5), 390-396 (2016).

15. Nickel N, Golpon H, Greer M et al. The prognostic impact of follow-up assessments in patients with idiopathic pulmonary arterial hypertension. Eur. Respir. J. 39(3), 589-596 (2012).

16. Al-Naamani N, Palevsky HI, Lederer DJ et al. Prognostic significance of biomarkers in pulmonary arterial hypertension. Ann. Am. Thorac Soc. 13(1), 25-30 (2016).

17. Rhodes CJ, Wharton J, Howard LS, Gibbs JS, Wilkins MR. Red cell distribution width outperforms other potential circulating biomarkers in predicting survival in idiopathic pulmonary arterial hypertension. Heart 97(13), 1054-1060 (2011).

18. Anwar A, Ruffenach G, Mahajan A, Eghbali M, Umar S. Novel biomarkers for pulmonary arterial hypertension. Respir. Res. 17(1), 88 (2016).

19. Jardim C, Souza R. Biomarkers and prognostic indicators in pulmonary arterial hypertension. Curr. Hypertens. Rep. 17(6), 556 (2015).

20. Lip GYH, Collet JP, Haude M et al. 2018 Joint European consensus document on the management of antithrombotic therapy in atrial fibrillation patients presenting with acute coronary syndrome and/or undergoing percutaneous cardiovascular interventions: a joint consensus document of the European Heart Rhythm Association (EHRA), European Society of Cardiology Working Group on Thrombosis, European Association of Percutaneous Cardiovascular Interventions (EAPCI), and European Association of Acute Cardiac Care (ACCA) endorsed by the Heart Rhythm Society (HRS), Asia-Pacific Heart Rhythm Society (APHRS), Latin America Heart Rhythm Society (LAHRS), and Cardiac Arrhythmia Society of Southern Africa (CASSA). Europace 21(2), 192-193 (2018).

21. D'andrea A, Naeije R, Grunig E et al. Echocardiography of the pulmonary circulation and right ventricular function: exploring the physiologic spectrum in 1480 normal subjects. Chest 145(5), 1071-1078 (2014). 